\title{
Risk Factors for Intraarticular Heterotopic Bone Formation in the Temporomandibular Joint in Juvenile Idiopathic Arthritis
}

\author{
Matthew L. Stoll, Dina Amin, Kathlyn K. Powell, Catherine H. Poholek, Rachel H. Strait, \\ Inmaculada Aban, Timothy Beukelman, Daniel W. Young, Randy Q. Cron, and Peter D. Waite
}

\begin{abstract}
Objective. Intraarticular corticosteroid (IAC) injections are often used to treat temporomandibular joint (TMJ) arthritis associated with juvenile idiopathic arthritis (JIA). One potential complication of IA therapy is heterotopic bone formation $(\mathrm{HBF})$. The purpose of our study was to evaluate risk factors for HBF development in children with JIA who received IA therapy for TMJ arthritis.

Methods. This was a retrospective study of children with JIA who had received $\geq 1$ IAC injection into the TMJ. Survival regression analysis was performed to identify risk factors for the development of HBF.

Results. There were 238 children included, of whom 33 (14\%) developed HBF. No cases of HBF were diagnosed prior to the initial injection. Univariate analysis revealed that the risk factors for development of HBF were the total number of injections received into the TMJ and age at diagnosis of JIA, while the length of time from diagnosis of JIA to the first injection was inversely associated with the risk of HBF formation. The total number of injections was no longer significant following adjusted survival models. Children with HBF had increased physical examination evidence of acute or chronic changes, namely decreased maximal incisal opening and increased likelihood of jaw deviation.

Conclusion. HBF within the TMJ is relatively common in patients with JIA receiving IAC injections for TMJ arthritis. Future prospective studies are required to delineate the risks posed by the injections themselves as opposed to the underlying disease activity, as well as to evaluate alternative forms of local therapy to the TMJ. (First Release May 15 2018; J Rheumatol 2018;45:1301-7; doi:10.3899/ jrheum.171306)
\end{abstract}

\section{Key Indexing Terms: \\ JUVENILE ARTHRITIS \\ INTRAARTICULAR INJECTIONS}

\section{TEMPOROMANDIBULAR JOINT HETEROTOPIC OSSIFICATION}

Juvenile idiopathic arthritis (JIA) is a chronic inflammatory disease of the joint lining tissue in childhood, with a prevalence in the United States of about 1 in 1000 children $^{1}$. Based on magnetic resonance imaging (MRI), temporomandibular

From the Department of Pediatrics at the University of Alabama at Birmingham, Birmingham, Alabama, USA.

M.L.Stoll, MD, PhD, MSCS, Department of Pediatric/Division of Rheumatology, University of Alabama at Birmingham (UAB); D. Amin, $D D S$, Department of Oral and Maxillofacial Surgery, UAB, and Department of Oral and Maxillofacial Surgery, Department of Surgery, Emory University School of Medicine; K.K. Powell, MD, Department of Oral and Maxillofacial Surgery, UAB; C.H. Poholek, MD, PhD,

Department of Medicine, UAB, and Department of Pediatrics, Children's Hospital of Pittsburgh at the University of Pittsburgh Medical Center; R.H. Strait, DMD, Department of Oral and Maxillofacial Surgery, UAB, and Department of Oral and Maxillofacial Surgery, Vanderbilt University Medical Center; I. Aban, PhD, Department of Biostatistics, UAB; T. Beukelman, MD, MSCE, Department of Pediatrics, UAB; D.W. Young, $M D$, Department of Radiology, UAB; R.Q. Cron, MD, PhD, Department of Pediatrics, UAB; P.D. Waite, MD, MPH, DDS, Department of Oral and Maxillofacial Surgery, $U A B$.

Address correspondence to Dr. M.L. Stoll, Associate Professor, Pediatric Rheumatology, University of Alabama at Birmingham, 16007 th Ave. South, Children's Park Place North Suite G10, Birmingham, Alabama 35233,USA.E-mail: MStoll@peds.uab.edu

Accepted for publication February 12, 2018. joint (TMJ) involvement occurs in 40-100\% of cases $2,3,4,5,6,7$. Undiagnosed and untreated, the expected morbidity of TMJ arthritis in children with JIA includes micrognathia, malocclusion, facial dysmorphism, and chronic pain ${ }^{8,9}$. Because TMJ arthritis can be active and destructive despite aggressive systemic therapy (conventional and biologic disease-modifying antirheumatic drug therapy; DMARD) and otherwise quiescent arthritis $6,10,11$, intraarticular (IA) corticosteroid (IACS) therapy has been used to control TMJ arthritis ${ }^{10}$. Several studies have documented the benefits of this approach ${ }^{7,12,13,14}$, although it is clearly not curative ${ }^{13,14,15}$. Although these studies have not reported significant adverse events beyond transient local Cushingoid reactions or subcutaneous atrophy and hypopigmentation ${ }^{10,16,17}$, longterm safety concerns have been raised ${ }^{18,19}$. In particular, 1 concern is the risk of heterotopic bone formation (HBF), defined as the presence of bone in soft tissue, such as within or immediately outside the TMJ capsule ${ }^{20}$. This can occur even in peripheral joints, where periarticular or IA calcifications have been reported following IACS injections in $0.2-6 \%$ of injected joints ${ }^{21,22,23}$. The risk may be higher in the TMJ because of unique anatomical features, including its small

Personal non-commercial use only. The Journal of Rheumatology Copyright @ ${ }^{2018}$. All rights reserved. 
space and IA ossification site ${ }^{24}$. A previous report documented the presence of $\mathrm{HBF}$ in 12 children with JIA who had undergone multiple injections with IACS, although it was unclear whether the primary risk factor in these subjects was the injections or the severity of the underlying arthritis that prompted local therapy ${ }^{20}$. Similarly, a second study reported IA ossifications in $21 \%$ of patients with JIA who had received IACS therapy to the TMJ ${ }^{25}$.

To evaluate this question further, clinical and imaging data were reviewed on a much larger cohort of 238 children with JIA who had undergone at least 1 round of IACS, with or without addition of IA infliximab (IAI) ${ }^{26}$. The subjects who developed HBF were compared to those who did not. This will help identify individuals at risk for HBF at the TMJ, as well as better define the pathophysiology of HBF in children with JIA.

\section{MATERIALS AND METHODS}

Subjects. This was a retrospective study of all children with JIA between the years 2009-2015 who were referred to a single oromaxillofacial (OMF) surgeon (PDW) for evaluation and management of TMJ arthritis, limited to those who underwent IACS injection of at least a single TMJ and had at least 1 followup MRI following the injection. All patients had been evaluated by a pediatric rheumatologist, and diagnosis of JIA was based upon the International League of Associations for Rheumatology criteria ${ }^{27}$. Demographic, clinical, and imaging data were obtained through review of the electronic medical records from Children's of Alabama $(\mathrm{CoA})$ and the University of Alabama at Birmingham (UAB). Maximal incisal opening (MIO) was routinely measured using the Therabite Measuring Scale (Atos Medical); there was no standardized protocol for its measurement, nor was there adjustment for the incisal vertical overbite. Presence versus absence of lateral deviation of the jaw at rest or with opening was documented at each visit; this was based solely on physical examination findings and did not take into account other potential causative factors (e.g., posterior crossbite).

Screening for TMJ arthritis. During the time of the study, most patients evaluated by pediatric rheumatology at CoA and diagnosed with JIA underwent routine screening for TMJ arthritis by MRI. The MRI protocol is as follows: prior to the administration of contrast, coronal T1-weighted (T1W) and fat-saturated (FS) T2W images are obtained, followed by FS sagittal proton density, FS T2W, and T1W images of the left and the right TMJ. Following the intravenous administration of Magnevist $0.1 \mathrm{mmol} / \mathrm{kg}$, coronal T1W images of the bilateral TMJ were obtained, followed by sagittal $\mathrm{T} 1 \mathrm{~W}$ images of the left and right TMJ. Children with abnormal findings on TMJ MRI were typically referred to a single OMF surgeon at UAB for evaluation and consideration for IA therapy for TMJ arthritis.

IA therapy for TMJ arthritis. All TMJ injections were performed by the same experienced OMF surgeon (PDW) at UAB. If patients failed systemic medical therapy and had 3 of 5 clinical criteria (pain, decreased maximal incisal opening, growth disturbance, open bite, MRI synovitis), they were considered for IA injections. The corticosteroid preparation used was either triamcinolone hexacetonide (TH) or triamcinolone acetonide (TA), depending on the availability of the former. The medicine was injected at $10 \mathrm{mg} / \mathrm{ml}$ (about $1 \mathrm{ml}$, sometimes less, depending on the joint space volume) up to twice per year ${ }^{13}$. Since late 2011, IAI was also offered to children with TMJ arthritis refractory to $\geq 1$ IACS injections ${ }^{26}$. This was injected at a concentration of $5 \mathrm{mg} / \mathrm{ml}$ (mixed 1:1 with $1 \%$ xylocaine), maximal volume possible, typically twice, occurring 6 weeks apart ${ }^{28}$. An OMF surgeon (PDW) with $>25$ years of experience performed the joint injections in the standard fashion of temporomandibular arthroscopic technique, which demonstrated a forward jaw thrust.
Screening for $H B F$. Children with substantial destructive changes on MRI (Figure 1), as well as concerning features by history or examination (e.g., abnormal motion and grinding), typically undergo traditional or cone beam computed tomography (CBCT) to evaluate for HBF. For our study, all CBCT scans were reviewed for evidence of HBF, namely lamellar bone within the soft tissue of the joint where bone does not normally exist. All suspected cases of HBF were confirmed by a pediatric radiologist (DWY). An illustration of an MRI suggestive of HBF is shown in Figure 1; a confirmatory CBCT scan is shown in Figure 2. Pathologic specimens obtained at time of joint replacement surgery in 2 patients are shown in Figure 3.

Statistical analysis. Data were summarized using means \pm SD for continuous variables and $\mathrm{n}(\%)$ for categorical data. The outcome of interest was the time to development of HBF following the JIA diagnosis. Those who did not develop HBF were classified as censored using their last MRI visit to compute the time to censor. Predictors and risk factors of interest were race, sex, JIA type, age at JIA diagnosis, time to first injection following JIA diagnosis, number of corticosteroid injections, number of infliximab injections, and number of total injections. To investigate the association of these

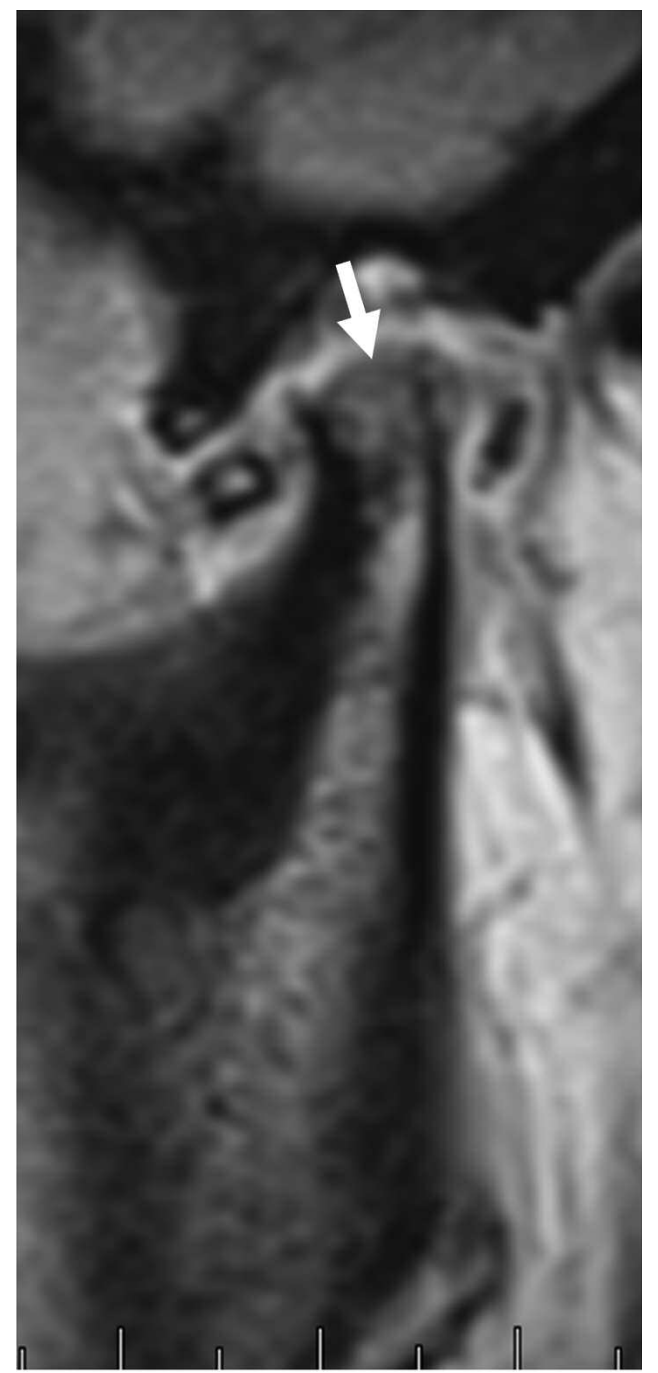

Figure 1. MRI image of sagittal T1W images of the right TMJ of a 19-year-old female with oligoarticular JIA. A narrowed joint space and erosive changes at the condylar head (arrow) are noted. MRI: magnetic resonance imaging; T1W: T1-weighted; TMJ: temporomandibular joint; JIA: juvenile idiopathic arthritis. 


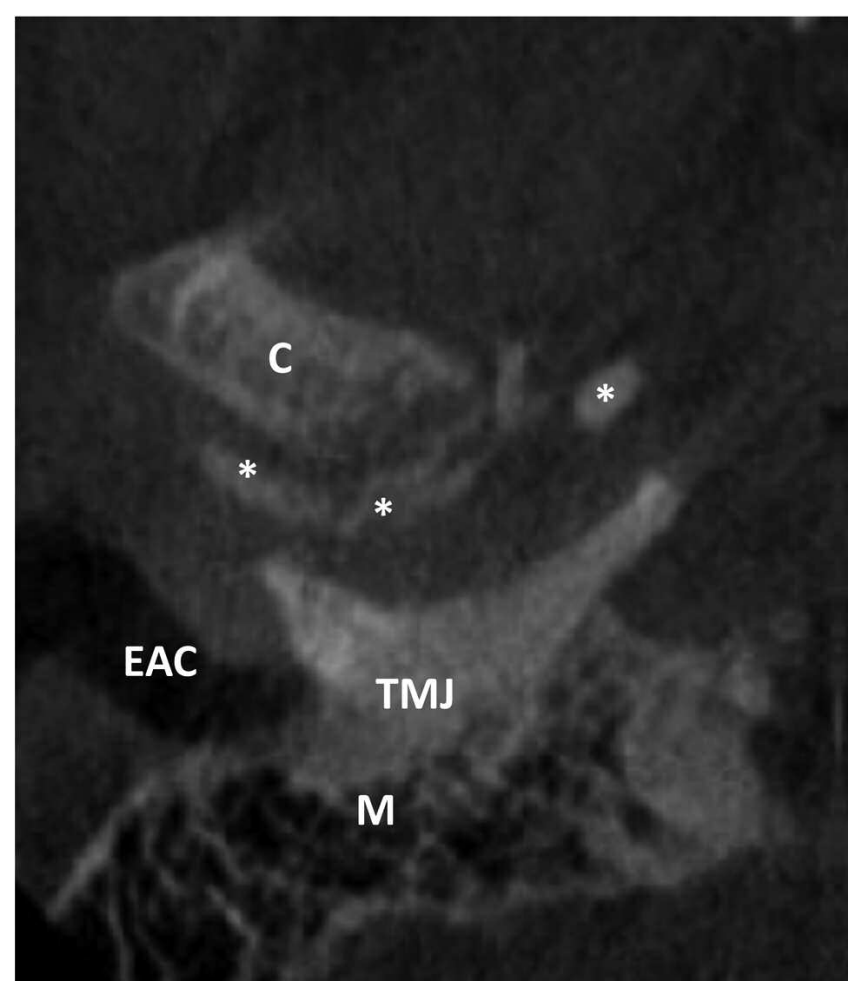

Figure 2. Axial CBCT image of the right TMJ of the same patient shown in Figure 1 , revealing bony fragments $(*)$ within the TMJ. The condylar head (C), external auditory canal (EAC), and mastoid air cells (M) are indicated. CBCT: cone beam computed tomography; TMJ: temporomandibular joint.

risk factors with the time to development of HBF, survival regression model, in particular Cox proportional hazard model, was fitted. The first step was to investigate the individual risk factors by fitting the Cox model separately for each factor. The second step was to fit the Cox model with all risk factors found to be significant in the first step to determine how that association changes after adjusting for other significant risk factors. Estimates of the HR and their CI were calculated; variables with $\mathrm{p}$ values $<0.05$ were deemed significant and included in the final adjusted model. Analyses were done using SAS version 9.4 (SAS Inc.). Comparisons of MIO and jaw deviation among children with and without HBF were performed with the Student $t$ test and chi-squared test, respectively.

Ethical statement. Approval for this study was obtained from the Institutional Review Board at UAB. Because of the retrospective features of this study, informed consent was granted an exemption in writing (IRB approval no. X111104004).

\section{RESULTS}

Patient population. There were 238 subjects included in the study; their clinical and demographic features are summarized in Table 1. As per the inclusion criteria, all the subjects had undergone at least 1 IACS injection; $55(23 \%)$ had also received IAI. In 233 (98\%), all the injections were bilateral. The durations of time from diagnosis of JIA to their final MRI, as well as from their first IACS injection to their final MRI, were $4.0 \pm 3.1$ and $2.1 \pm 1.3$ years, respectively (Table 1). Most of the children received traditional DMARD in conjunction with systemic biologic therapy.

Predictors of $H B F$. There were 33 subjects (13.9\%) who developed HBF (Figure 3); this was present unilaterally on the right in $16(48 \%)$, unilaterally on the left in $5(15 \%)$, and bilaterally in $12(36 \%)$. Table 2 shows predictors of HBF by Cox model, which is a form of survival analysis. TH and TA were grouped together under CS, because of the rarity of the latter during the time period of the study. In the unadjusted modeling of time to HBF following JIA diagnosis, the only factors found to be associated with increased risk of HBF were age at diagnosis of JIA and the total number of injections. In this case, the hazard of developing HBF from JIA diagnosis increased by $28 \%$ for every year increase in the age at diagnosis (HR 1.279, 95\% CI 1.169-1.398, p < 0.0001)
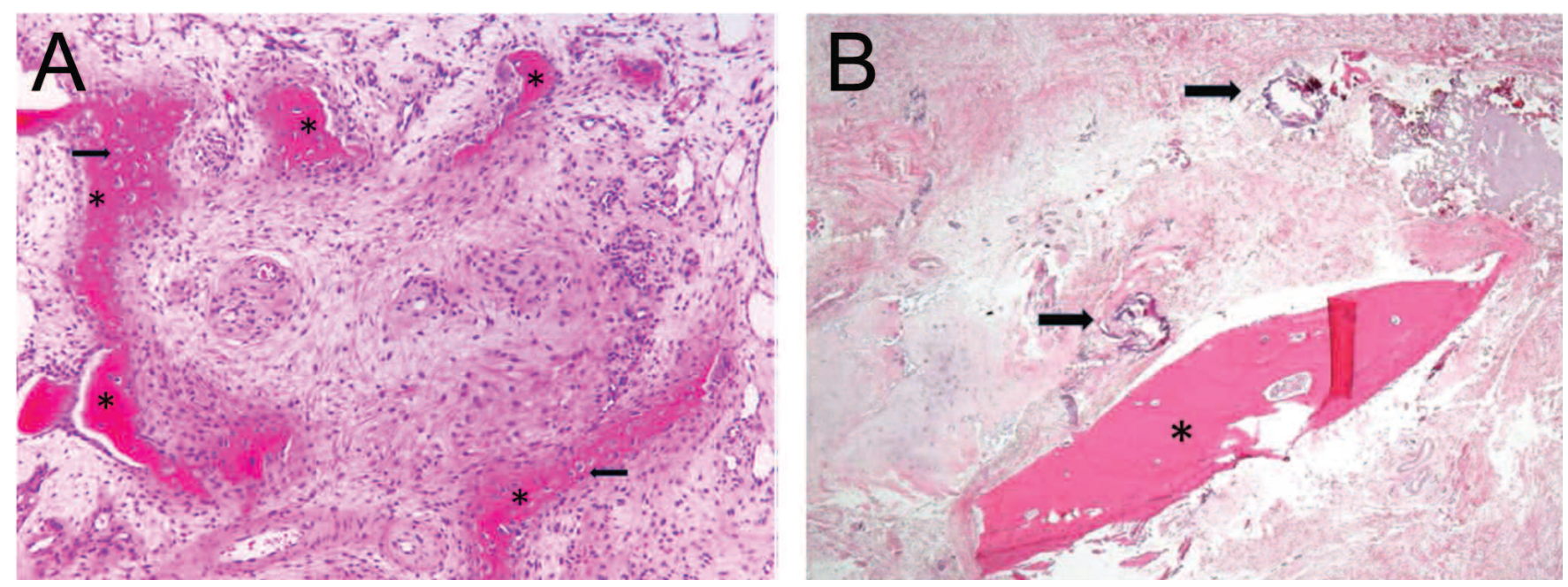

Figure 3. Pathology slides showing new bone formation in patients with HFB. A. Condylar biopsy of a 14-year-old female with oligoarticular JIA. New bone formation (asterisks) with eosinophilic osteoid and osteocytes within lacunar spaces (arrows) is observed in an arc surrounded by loosely arranged reactive stroma (H\&E, 33×). B. Condylar biopsy of a 22-year-old female with oligoarticular JIA. A bony spicule (*) is embedded within the connective tissue. Two small areas of non-bony calcification are also observed (arrows; H\&E, 4×). HFB: heterotopic bone formation; JIA: juvenile idiopathic arthritis.

Personal non-commercial use only. The Journal of Rheumatology Copyright @ 2018 . All rights reserved. 
Table 1. Clinical and demographic features of subjects included in this study. Values are $\mathrm{n}(\%)$ or mean \pm SD unless otherwise specified.

\begin{tabular}{|c|c|}
\hline Feature & Result \\
\hline $\mathrm{N}$ & 238 \\
\hline Female & $174(73)$ \\
\hline \multicolumn{2}{|l|}{ Race/ethnicity } \\
\hline Hispanic & $3(1.3)$ \\
\hline White & $204(86)$ \\
\hline African American & $30(13)$ \\
\hline Mixed & $1(0.4)$ \\
\hline \multicolumn{2}{|l|}{ Category } \\
\hline Oligoarticular & $114(48)$ \\
\hline $\mathrm{RF}$ - polyarticular & $37(16)$ \\
\hline $\mathrm{RF}+$ polyarticular & $11(4.6)$ \\
\hline Psoriatic JIA & $27(11)$ \\
\hline Enthesitis-related arthritis & $43(18)$ \\
\hline Systemic JIA & $6(2.5)$ \\
\hline Age at final MRI & $13.2 \pm 4.2$ \\
\hline Duration since JIA diagnosis, yrs & $4.0 \pm 3.1$ \\
\hline Duration since first IACS, yrs & $2.1 \pm 1.3$ \\
\hline \multicolumn{2}{|l|}{ No. IACS } \\
\hline 1 & $114(48)$ \\
\hline 2 & $88(37)^{*}$ \\
\hline 3 & $30(13)^{* *}$ \\
\hline$\geq 4$ & $6(2.5)$ \\
\hline \multicolumn{2}{|l|}{ No. IAI } \\
\hline 0 & $183(77)$ \\
\hline 1 & $15(6.3)$ \\
\hline 2 & $26(11)$ \\
\hline 3 & $6(2.5)$ \\
\hline$\geq 4$ & $8(3.3)$ \\
\hline \multicolumn{2}{|l|}{ Systemic medications } \\
\hline None & $10(4.2)$ \\
\hline cDMARD only & $15(6.3)$ \\
\hline bDMARD \pm cDMARD & $213(90)$ \\
\hline IL- 1 or IL- 6 antagonist & $6(2.5)$ \\
\hline TNFi & $209(88)$ \\
\hline $\mathrm{ABA}$ & $19(8.0)$ \\
\hline RTX & $5(2.1)$ \\
\hline
\end{tabular}

*Of the 88 subjects who had 2 rounds of IACS, treatment was bilateral in $86 ; 1$ subject underwent unilateral injection once and the other received unilateral injections on both occasions. ** Of the 26 subjects who had 3 rounds of IACS, 2 had unilateral injections on 2 occasions, and 1 had a unilateral injection on 1 occasion. All IAI injections were bilateral. RF: rheumatoid factor; JIA: juvenile idiopathic arthritis; MRI: magnetic resonance imaging; IACS: intraarticular corticosteroids; IAI: intraarticular infliximab; cDMARD: conventional disease-modifying antirheumatic drug (hydroxychloroquine, leflunomide, methotrexate, mycophenolate mofetil, sulfasalazine); bDMARD: biologic DMARD; IL-1: interleukin 1; TNFi: tumor necrosis factor inhibitors; ABA: abatacept; RTX: rituximab.

and $25 \%$ for every additional injection (HR $1.254,95 \%$ CI $1.04-1.512, \mathrm{p}=0.0184)$. In general, children with more severe arthritis were more likely to receive repeated IA TMJ injections, and it is our practice to screen for TMJ arthritis early in the disease course and to refer for injections if necessary. However, 43 of the patients reported herein had delays of upwards of 3 years from diagnosis of JIA to initial diagnosis of TMJ arthritis. In 36 of these patients (84\%), this delay was because the children were either diagnosed with
JIA prior to the establishment of a pediatric rheumatology program at CoA in 2007, or because the children transferred their care from other providers. Although there is no way to know the actual duration of TMJ arthritis in these patients prior to the initial assessment, since TMJ arthritis is frequently present at disease onset ${ }^{2}$, it is likely that many of them had longstanding TMJ arthritis. To the extent that active TMJ arthritis is a risk factor for HBF, it would follow that these subjects would be at greatest risk. Therefore, a variable representing the time from diagnosis of JIA to the initial intraTMJ CS injection was created. Of interest, the time to first injection showed evidence of a protective effect. In particular, for every additional year added to the time to first injection after JIA diagnosis, the hazard of HBF was estimated to decrease by $56 \%$ (HR $0.44,95 \%$ CI $0.296-0.655$ ). Sex, race, and JIA category had no evident association with the development of HBF from JIA diagnosis. In the adjusted model with age of JIA diagnosis, total number of injections, and time to first injection from JIA diagnosis (Table 2), the total number of injections was no longer a significant predictor; however, age at JIA diagnosis and time to first injection from diagnosis were still significant risk factors. HR were similar for these risk factors in both the unadjusted and adjusted models.

As a sensitivity analysis, the analysis was repeated excluding 18 subjects who were initially treated at an outlying hospital, for whom there might be less precision regarding the date of diagnosis of JIA. This did not dramatically change the final model, which again showed that time from diagnosis to the first injection was protective with $\mathrm{HR}$ of 0.55 (95\% CI $0.36-0.82, \mathrm{p}=0.0075)$, and the age at JIA diagnosis was associated with increased risk, with HR of 1.2 (95\% CI 1.09-1.32, $\mathrm{p}=0.0002)$.

Regarding potential differences between local therapies, only 20 patients received therapy with TA, of whom 13 additionally received $\mathrm{TH}$, so we were likely to be insufficiently powered to compare these 2 CS preparations. Regarding IAI, every patient who received this therapy had also received 1 or more corticosteroid injections, so we could not compare IAI with IACS. After adjusting for the total number of injections, there did not appear to be a difference in type (i.e., CS vs infliximab), but we were underpowered to detect such an effect.

Outcome of $\mathrm{HBF}$. Information on $\mathrm{MIO}$ and presence versus absence of jaw deviation at the time of the final visit were available on 223 and all 238 patients, respectively (Table 3). Despite younger age, children without HBF had larger MIO $(4.5 \pm 0.73$ vs $3.9 \pm 1.0, \mathrm{p}=0.004)$ and were significantly less likely to have presence of jaw deviation on opening (28\% vs $47 \%, p=0.034)$. Finally, 3 of the 33 subjects who developed $\mathrm{HBF}$ required joint replacement surgery.

\section{DISCUSSION}

Findings of HBF following TMJ IA therapy raise the possibility that they represent sequelae of the injection itself.

Personal non-commercial use only. The Journal of Rheumatology Copyright @ 2018. All rights reserved 
Table 2. Cox regression analysis of children with versus without HBF.

\begin{tabular}{lcc}
\hline Predictor & Unadjusted HR $(95 \% \mathrm{CI})$ & Final model HR (95\% CI) \\
\hline Race, white vs other & 0.6135 & Not included \\
Sex & 0.7712 & Not included \\
JIA category & 0.5182 & Not included \\
Age at JIA diagnosis, yrs & $\mathbf{1 . 2 8}(\mathbf{1 . 1 7 - 1 . 4 0 )}, \mathbf{p}<\mathbf{0 . 0 0 0 1}$ & $\mathbf{1 . 1 9}(\mathbf{1 . 0 8}-\mathbf{1 . 3 0}), \mathbf{p}=\mathbf{0 . 0 0 0 2}$ \\
Time to first injection following & $\mathbf{0 . 4 4 1}(\mathbf{0 . 3 0 - 0 . 6 6 )}, \mathbf{p}<\mathbf{0 . 0 0 0 1}$ & $\mathbf{0 . 5 0}(\mathbf{0 . 3 4 - 0 . 7 4 )}, \mathbf{p}=\mathbf{0 . 0 0 0 5}$ \\
$\quad$ diagnosis of JIA & 0.3588 & Not included \\
No. IACS injections & 0.0519 & Not included \\
No. IAI & $\mathbf{1 . 2 5}(\mathbf{1 . 0 4 - 1 . 5 1 )}, \mathbf{p}=\mathbf{0 . 0 1 8 4}$ & $\mathbf{1 . 1 1 8}(\mathbf{0 . 9 0 - 1 . 4 0}), \mathrm{p}=0.3263$ \\
Total IA injections &
\end{tabular}

Values in bold face are statistically significant. HBF: heterotopic bone formation; JIA: juvenile idiopathic arthritis; IA: intraarticular; IACS: IA corticosteroids, IAI: IA infliximab.

Table 3. HBF outcomes.

\begin{tabular}{lccc}
\hline Feature & \multicolumn{2}{c}{ HBF } & p \\
& Present & Absent & \\
\hline MIO*, n; mean \pm SD & $31 ; 3.9 \pm 1.0$ & $192 ; 4.5 \pm 0.73$ & $\mathbf{0 . 0 0 4}$ \\
Jaw deviation**, n (\%) & $15 / 32(47 \%)$ & $58 / 205(28 \%)$ & $\mathbf{0 . 0 3 4}$
\end{tabular}

$* \mathrm{n}=223 ; * * \mathrm{n}=238$. Data on MIO and jaw deviation were missing from 17 and 2 subjects, respectively. Values in bold face are statistically significant. HBF: heterotopic bone formation; MIO: maximal incisal opening.

Indeed, findings such as this have prompted some to limit the use of IACS into the TMJ ${ }^{18,19}$. However, a study from Seattle of subjects with HBF noted that of the 2 subjects who underwent biopsy, both had active new bone formation suggestive of sequelae of persistent arthritis, rather than crystalline deposition as would be expected if the etiology were the CS injections themselves ${ }^{20}$. The data presented herein provides mixed conclusions as to the etiology of HBF. Clearly, the number of injections was associated with risk of HBF. However, the challenge of studying risk factors for HBF is that for obvious ethical reasons, healthy children or even JIA patients without known TMJ arthritis do not undergo IA therapy. Thus, study of the total number of injections is confounded by the disease severity that prompted these injections. An important finding in our work is that children who had a long lag between diagnosis of JIA and introduction of IA therapy appeared to be protected against the development of HBF. If unopposed arthritis in the joint were the main factor, the exact opposite finding would have been anticipated; thus, our data suggest that the injections themselves may be an important contributing factor to the development of HBF, conclusions also reached by Lochbuhler, et $a l^{25}$. If this in indeed the case, it is unclear whether this increased risk of HBF associated with IA therapy of the TMJ is limited to CS or associated with any therapy; in our study, there was no obvious protective benefit associated with infliximab, although we may have lacked sufficient power to detect such an effect. Nevertheless, this finding, in combination with our previous data showing that there is no evident effectiveness of IAI ${ }^{26}$, fails to provide any compelling rationale to switch from IACS to IAI. Further, to the extent that any IA therapy may predispose to HBF, it follows that simple lavage may also predispose patients to this complication, although none of the patients were treated in that manner during the study period.

Patients with HBF had a smaller MIO despite increased age and increased likelihood of jaw deviation, both of which findings are associated with increased severity of TMJ arthritis ${ }^{29,30}$. This may simply reflect complications of the worsening arthritis that prompted repeat IA therapy. It is also possible that $\mathrm{HBF}$ itself may worsen the severity of the arthritis. Indeed, the bony fragments are visible within the joint space (Figure 2 and Figure 3 ) and thus may irritate the synovial lining. In support of this possibility, data published in 1984 suggested that periarticular ossifications following IA therapy are composed of hydroxyapatite crystals that can produce inflammatory arthritis ${ }^{31}$, in which case the therapy could indirectly cause worsening of the underlying disease. As noted above, 3 children underwent joint replacement therapy of the TMJ, indicative of a severely damaged joint.

An unexpected finding was that HBF was more likely in older children. Specifically, each additional year at diagnosis of JIA was associated with a $38 \%$ increase in the hazard of HBF (Table 2). This does not appear to reflect differences within the risk of JIA categories or by sex, none of which were statistically significant. Instead, it suggests that older children may have greater intrinsic risk, perhaps because injections were being given during the growth spurt, and thus aggressive systemic therapy may be preferable over local therapy in this population.

Our study has limitations. This was a retrospective study, without any specific protocols for IA therapy, repeat MRI, or $\mathrm{CBCT}$ scans. Because of the features of the findings, CBCT scan is clearly far more sensitive for the detection of $\mathrm{HBF}$ as compared to MRI, so it is possible that cases were missed. However, all the subjects included in the study did have at least 1 followup MRI following administration of IA therapy, and it is our practice to obtain CBCT scans on those with

Personal non-commercial use only. The Journal of Rheumatology Copyright (C) 2018. All rights reserved. 
particularly destructive changes. The MIO may be challenging to measure, particularly in uncooperative children or in patients with overbite or who are missing their incisors. Despite these limitations, however, it is widely used as an outcome measure in the assessment of TMJ arthritis $2,10,17$.

Finally, it bears emphasis that HBF may not by itself affect function of the TMJ. In contrast, there is an extensive body of literature indicating that active TMJ arthritis can result in altered shape and function, complicating activities of daily life including mastication and talking ${ }^{7}$. Further, it is clear that systemic therapies do not always treat the underlying arthritis ${ }^{6}$. Therefore, while we agree with previous calls for caution in the use of IACS of the TMJ ${ }^{18,19}$, these risks must be weighed against the risks of unopposed arthritis involving this joint, particularly in patients with substantial arthritic changes in whom systemic therapy has already optimized. Additionally, future studies should prospectively evaluate the safety and effectiveness of IACS, as compared to systemic therapy alone, $\mathrm{IAI}^{26}$, arthrocentesis with lavage only ${ }^{32}$, and possibly even iontophoresis ${ }^{33}$.

\section{ACKNOWLEDGMENT}

The authors acknowledge Drs. Thomas Winokur and David Kelly for assistance with creating the pathology figures.

\section{REFERENCES}

1. Gewanter HL, Roghmann KJ, Baum J. The prevalence of juvenile arthritis. Arthritis Rheum 1983;26:599-603.

2. Weiss PF, Arabshahi B, Johnson A, Bilaniuk LT, Zarnow D, Cahill AM, et al. High prevalence of temporomandibular joint arthritis at disease onset in children with juvenile idiopathic arthritis, as detected by magnetic resonance imaging but not by ultrasound. Arthritis Rheum 2008;58:1189-96.

3. Abramowicz S, Cheon JE, Kim S, Bacic J, Lee EY. Magnetic resonance imaging of temporomandibular joints in children with arthritis. J Oral Maxillofac Surg 2011;69:2321-8.

4. Cannizzaro E, Schroeder S, Muller LM, Kellenberger CJ, Saurenmann RK. Temporomandibular joint involvement in children with juvenile idiopathic arthritis. J Rheumatol 2011;38:510-5.

5. Cedstromer AL, Ahlqwist M, Andlin-Sobocki A, Berntson L, Hedenberg-Magnusson B, Dahlstrom L. Temporomandibular condylar alterations in juvenile idiopathic arthritis most common in longitudinally severe disease despite medical treatment. Pediatr Rheumatol Online J 2014;12:43.

6. Stoll ML, Sharpe T, Beukelman T, Good J, Young D, Cron RQ. Risk factors for temporomandibular joint arthritis in children with juvenile idiopathic arthritis. J Rheumatol 2012;39:1880-7.

7. Ringold $\mathrm{S}, \mathrm{Cron} \mathrm{RQ}$. The temporomandibular joint in juvenile idiopathic arthritis: frequently used and frequently arthritic. Pediatr Rheumatol Online J 2009;7:11.

8. Stabrun AE. Impaired mandibular growth and micrognathic development in children with juvenile rheumatoid arthritis. A longitudinal study of lateral cephalographs. Eur J Orthod 1991;13:423-34.

9. Larheim TA, Hoyeraal HM, Stabrun AE, Haanaes HR. The temporomandibular joint in juvenile rheumatoid arthritis. Radiographic changes related to clinical and laboratory parameters in 100 children. Scand J Rheumatol 1982;11:5-12.

10. Arabshahi B, Dewitt EM, Cahill AM, Kaye RD, Baskin KM, Towbin RB, et al. Utility of corticosteroid injection for temporomandibular arthritis in children with juvenile idiopathic arthritis. Arthritis Rheum 2005;52:3563-9.

11. Knights D, Silverberg MS, Weersma RK, Gevers D, Dijkstra G, Huang $\mathrm{H}$, et al. Complex host genetics influence the microbiome in inflammatory bowel disease. Genome Med 2014;6:107.

12. Habibi S, Ellis J, Strike H, Ramanan AV. Safety and efficacy of US-guided CS injection into temporomandibular joints in children with active JIA. Rheumatology 2012;51:874-7.

13. Stoll ML, Good J, Sharpe T, Beukelman T, Young D, Waite PD, et al. Intra-articular corticosteroid injections to the temporomandibular joints are safe and appear to be effective therapy in children with juvenile idiopathic arthritis. J Oral Maxillofac Surg 2012;70:1802-7.

14. Resnick CM, Vakilian PM, Kaban LB, Peacock ZS. Quantifying the effect of temporomandibular joint intra-articular steroid injection on synovial enhancement in juvenile idiopathic arthritis. J Oral Maxillofac Surg 2016;74:2363-9.

15. Stoustrup P, Kristensen KD, Kuseler A, Pedersen TK, Herlin T. Temporomandibular joint steroid injections in patients with juvenile idiopathic arthritis: an observational pilot study on the long-term effect on signs and symptoms. Pediatr Rheumatol Online J 2015;13:62.

16. Hugle B, Laxer RM. Lipoatrophy resulting from steroid injection into the temporomandibular joint. Arthritis Rheum 2009;60:3512.

17. Ringold S, Torgerson TR, Egbert MA, Wallace CA. Intraarticular corticosteroid injections of the temporomandibular joint in juvenile idiopathic arthritis. J Rheumatol 2008;35:1157-64.

18. Stoustrup P, Kristensen KD, Verna C, Kuseler A, Pedersen TK, Herlin T. Intra-articular steroid injection for temporomandibular joint arthritis in juvenile idiopathic arthritis: a systematic review on efficacy and safety. Semin Arthritis Rheum 2013 ;43:63-70.

19. Stoustrup P, Twilt M. Therapy. Intra-articular steroids for TMJ arthritis - caution needed. Nat Rev Rheumatol 2015;11:566-7.

20. Ringold S, Thapa M, Shaw EA, Wallace CA. Heterotopic ossification of the temporomandibular joint in juvenile idiopathic arthritis. J Rheumatol 2011;38:1423-8.

21. Breit W, Frosch M, Meyer U, Heinecke A, Ganser G. A subgroup-specific evaluation of the efficacy of intraarticular triamcinolone hexacetonide in juvenile chronic arthritis. J Rheumatol 2000;27:2696-702.

22. Job-Deslandre C, Menkes CJ. Complications of intra-articular injections of triamcinolone hexacetonide in chronic arthritis in children. Clin Exp Rheumatol 1990;8:413-6.

23. Sparling M, Malleson P, Wood B, Petty R. Radiographic followup of joints injected with triamcinolone hexacetonide for the management of childhood arthritis. Arthritis Rheum. 1990;33:821-6

24. Arabshahi B, Cron RQ. Temporomandibular joint arthritis in juvenile idiopathic arthritis: the forgotten joint. Curr Opin Rheumatol 2006; 18:490-5.

25. Lochbuhler N, Saurenmann RK, Muller L, Kellenberger CJ. Magnetic resonance imaging assessment of temporomandibular joint involvement and mandibular growth following corticosteroid injection in juvenile idiopathic arthritis. J Rheumatol 2015; 42:1514-22.

26. Stoll ML, Vaid YN, Guleria S, Beukelman T, Waite PD, Cron RQ. Magnetic resonance imaging findings following intraarticular infliximab therapy for refractory temporomandibular joint arthritis among children with juvenile idiopathic arthritis. J Rheumatol 2015;42:2155-9.

27. Petty RE, Southwood TR, Manners P, Baum J, Glass DN, Goldenberg J, et al; International League of Associations for Rheumatology. International League of Associations for Rheumatology classification of juvenile idiopathic arthritis: second revision, Edmonton, 2001. J Rheumatol 2004;31:390-2.

28. Stoll ML, Morlandt AB, Teerawattanapong S, Young D, Waite PD, Cron RQ. Safety and efficacy of intra-articular infliximab therapy

Personal non-commercial use only. The Journal of Rheumatology Copyright $\odot$ 2018. All rights reserved. 
for treatment-resistant temporomandibular joint arthritis in children: a retrospective study. Rheumatology 2013;52:554-9.

29. Pedersen TK, Kuseler A, Gelineck J, Herlin T. A prospective study of magnetic resonance and radiographic imaging in relation to symptoms and clinical findings of the temporomandibular joint in children with juvenile idiopathic arthritis. J Rheumatol 2008;35:1668-75.

30. Ronchezel MV, Hilario MO, Goldenberg J, Lederman HM, Faltin K Jr., de Azevedo MF, et al. Temporomandibular joint and mandibular growth alterations in patients with juvenile rheumatoid arthritis. J Rheumatol 1995;22:1956-61.
31. Dalinka MK, Stewart V, Bomalaski JS, Halpern M, Kricun ME. Periarticular calcifications in association with intra-articular corticosteroid injections. Radiology 1984;153:615-8.

32. Olsen-Bergem H, Bjornland T. A cohort study of patients with juvenile idiopathic arthritis and arthritis of the temporomandibular joint: outcome of arthrocentesis with and without the use of steroids. Int J Oral Maxillofac Surg 2014;43:990-5.

33. Mina R, Melson P, Powell S, Rao M, Hinze C, Passo M, et al. Effectiveness of dexamethasone iontophoresis for temporomandibular joint involvement in juvenile idiopathic arthritis. Arthritis Care Res 2011;63:1511-6. 